\title{
The Practices of Critical Thinking Component and Its Impact in Malaysian Nurses Health Education
}

\author{
Abdul Ghani Kanesan Abdullah \\ School of Educational Studies Universit Sains Malaysia \\ Penang 11800 Malaysia \\ Tel: 60-4-653-4328Ｅ-mail: agk@usm.my \\ Naser Jamil Alzaidiyeen \\ School of Educational Studies Universit Sains Malaysia \\ Penang 11800 Malaysia
}

Tel: 60-12-498-9384Ｅ-mail: naser_jamel@yahoo.com

$\mathrm{Ng}$ Mooi Yee

School of Educational Studies Universit Sains Malaysia

Penang 11800 Malaysia)

Tel: 60-16-454-4506Ｅ-mail: Mooi@yahoo.com

\begin{abstract}
The purpose of this research is to study the impact of the critical thinking component in the health education curriculum of nurses for patients with different health needs. Data for this research was gathered from mixed approaches, quantitative and qualitative approaches. For the quantitative approach 84 student nurses were selected randomly to represent the experiment and control groups in a private medical college in Northern part of Pennisular Malaysia. The 2 groups of students had been exposed to health education and clinical training in the wards as determined by the nursing curriculum. Following that, a treatment in the form of a critical thinking module which consisted of critical thinking related activities, was only given to the experiment group. Following the quantitative session, the qualitative approach was used. In this session, 5 student nurses were selected randomly and they were interviewed for 2 times, the first session was after the treatment was given and the second session was after clinical training with the patients. Besides these interviews, the patients concerned were also interviewed Findings from t-test and ANCOVA showed significant difference in the achievement between the experiment and control groups. In other words, these findings showed that there was a significant impact of the critical thinking component in the health education curriculum of nurses. Qualitative data findings showed that the respondents demonstrated thinking skills during their clinical training. Their patients too voiced individual views and perceptions. The students also felt that their thinking had improved after their induction to the critical thinking module.
\end{abstract}

Keywords: Critical Thinking, Curriculum, Nurses, Health Education, Patients, Decision-making

\section{Introduction}

The Ministry of Health and the Nursing Board of Malaysia had realized the importance of health education and had integrated the teaching of health education into the basic nursing curriculum (Nurse Curriculum, 1996). However, the focus of health education in this basic nursing curriculum is only in the community health nursing discipline where 14 hours have been allocated for the imparting of knowledge on health education. Therefore, health education is only touched upon as part of general nursing interventions in the other nursing disciplines on a "touch and go" basis. The theory content of health education especially on patient survival skills such as injection techniques and diet education is given more focus. Thus, the impact of the health education delivered was compromised without due consideration for the patient in his reality setting.

\section{Background of the Study}

Today's patients are becoming better consumers of health care. With internet access patients are becoming responsible for their own health maintenance, modifying their behaviour and managing chronic diseases with complex therapeutic regimens (Rankin, Stallings \& London, 2005). Patients demand to know more about their health conditions and 
treatment through health education as they know it is their right to participate in decision-making regarding their own health care.

Generally nurses too seek to deliver holistic care for their patients. However, where the delivery of health education in nursing practice is concerned, much needs to be improved. The general notion nurses have is that if they have done their job of informing their patients and their families about the essentials of staying alive and away from hospital, they have carried out health education successfully (Rankin \& Stallings, 1990). There is usually no follow-up on the effectiveness of the health education given. Its impact is only known when the patient is readmitted for the same complaint or when his or her disease condition has deteriorated and complications have set in.

This scenario is evident in a study carried out by Ruzlan, Hairne \& Nurulaidah (2006) in Malaysia revealed that even though health education was given as a matter of routine, the emphasis on its importance did not seem to get communicated to the respondents. In a survey on home care carried out by Liang (2007) it was found that respondents and their families were not given adequate or clear information which they could understand pertaining to their after care at home. It was also found that they generally preferred health education to be given to them by the nurses in their own home settings rather than in the hospital set-up.

Considering the above factors related to the current health education management, it indicated that much needs to be carried out to improve the quality of health education management. To begin with, before the nurse can carry out effective nursing functions including health education delivery, they have to be equipped and trained with the appropriate knowledge, behaviours and attitudes in order to promote health, help prevent diseases and care for the sick through nursing education (Document 3, Detailed Educational Programme, 2004). The students are not taught how to assess and think critically to deliver health talks tailor-made for patients with different needs. Besides this, there is also the critical lack of experienced and trained nurse educators (Chua, 2004). Once student nurses had completed their theory component, they were expected to know how to transfer theory into practice by delivering it effectively to their patients and their families as each situation arose. Bandura (1989) had proposed that individuals learn from observing and imitating a model's behaviour. Therefore, the student nurses would have learnt their clinical nursing skills through observing role models in their nurse teachers and trained staff nurses in the clinical settings.

Besides the teaching learning process, other vicarious aspects could be contributory to the ineffectiveness of health education outcomes. If the health education carried out is ineffective, one would have to reconsider the way it is being communicated to the patients. Barriers to effective communication could have occurred between sender and recipient taking into consideration the messages conveyed as well as the communication channels used (Kozier, Erb, Berman \& Snyder, 2004). Other aspects include patient understanding of the health education content which could be due to differing perceptions since they are individuals from different socio-cultural backgrounds. Since nurses are usually hard pressed for time to give proper health education, they tend to concentrate only on survival skills such as injection techniques, dietary requirements and signs and symptoms of disease recurrences. The patient's reality problems, reinforcement and evaluation of learning goals are often overlooked.

\section{Application of Critical Thinking in Nursing Practice}

The traditional general thinking skills may be used to analyze, judge and argue on an issue. These skills may be adequate in dealing with standard situations and solutions. However, critical thinking and decision-making have been associated with improved clinical expertise as critical thinking is the centre of the process of clinical reasoning and clinical judgement (Jackson, 2004; Martin, 2000).

Using critical thinking to develop a plan of nursing care requires considering human factors that might influence the care plan as nurses interact with patients, families, and community as well as other health care providers in the process of providing appropriate, individualized nursing care. The culture, attitudes and thought processes of the patients, nurses and others affect the critical thinking process throughout the nurse-patient interactions (Wilkinson, 2001).

Nurses must use critical thinking skills in all practice settings. Regardless of the setting, each patient situation is viewed as unique and dynamic. The unique factors that patients and nurses bring to the health care situation are considered, studied, analyzed and interpreted. Interpretation of the information allows the nurse to focus on those factors that are most relevant and significant to the clinical situation. Decisions about what to do and how to do it are developed into a plan of action. These skills include systematic and comprehensive assessment, recognition of assumptions and inconsistencies, verification of reliability and accuracy, identification of missing information, distinguishing relevant from irrelevant information, support of the evidence with facts and conclusions, priority setting with timely decision-making determination of patient-specific outcomes and reassessment of responses and outcomes (Alfaro-LeFavre, 2003).

In the area of health education, in order to be effective, the student nurse has to apply his or her thinking processes in order to be able to manage each of his or her patients according to the individual patient setting and requirements. Critical thinking is a higher-order thinking process which uses basic thinking processes to analyze arguments and 
generate insight into particular meanings and interpretations, develop cohesive, logical reasoning patterns, understand assumptions and biases underlying particular positions and attain a credible, concise, and convincing style of presentation to put forward an argument (Paul, 1993).

\section{Critical Thinking Skills in Nursing}

In nursing, LeMone and Burke (2008) categorized critical thinking skills into divergent thinking which is the ability to weigh the the importance of information gathered in order to explore alternatives and draw conclusions from the relevant data collected. Being able to reason is another thinking skill that is important in that the nurse is able to discriminate between facts and non - facts so that decisions are made in a systematic, logical manner to solve problems. The ability to clarify similarities and differences from irrelevant information will also help the nurse focus on the situation at hand. Reflection is when the nurse takes time to think and compare different situations with similar solutions. This reflective exercise in turn helps to give the nurse options and alternatives when caring for different patients. However, this reflection cannot take place in an emergency situation since the nurse needs to follow standard protocols in such situations.

Though the nurse may know "the what" of thinking but from the nursing process, they also has to know "the how" of thinking that is the thinking skills required for a specific situation (Kozier, Erb, Berman \& Snyder, 2004). Critical thinking skills tools could be used for this purpose and de-Bono (1999) believes that thinking skills can be taught. This consideration has resulted in the development of thinking tools, among which is the CoRT 1 thinking method (de-Bono, 1972). This method focuses on different aspects of thinking from which thinking tools are developed and taught. These thinking tools are as follow:

\subsection{P.M.I (Plus, Minus, Interesting)}

This is a tool that permits a non-dialectic exploration of an idea by adding a third, value-free category to the usual "pros and cons' approach to decision-making. It focuses one's full attention and energy equally and deliberately to all the positive, negative and interesting sides of an issue without any preconceived notions or prejudices, thus helping to avoid immediate acceptance or rejection of an idea or issue. A course of action can then be selected from a range of options provided from considering the P.M.I. aspects of the issue concerned.

\subsection{C.A.F. (Consider All Factors)}

This technique involves the thinking of all the factors involved in a particular situation. Care should be taken to avoid only considering those first factors that come to mind. By doing a C.A.F. one gathers as much information as is possible in exploring a situation from all angles, evaluates and then considers it before a decision is made.

\subsection{O.P.V. (Other People's Views)_}

This tool helps one to focus consciously and deliberately at other people's viewpoints to better assess the impact of a proposed decision. To be able to listen to other people's views also helps to increase one's sensitivity to the opinions of others so that their views are considered during the decision-making process of an issue.

\subsection{F.I.P. (First Important Priorities)}

This strategy is used when one has to focus and select priorities before a decision is made. Therefore, the most important ideas are considered first in the decision-making process. However, this method requires one to make judgement on a situation as what is important for one individual may not be so for another person.

\subsection{A.G.O. (Aims, Goals, Objectives)}

This tool aims to provide the general direction towards a final destination of a goal while objectives are recognizable points of achievements along the way towards that goal. This strategy helps one to focus directly and deliberately on one's own intention and the intention of others behind an action.

\subsection{A.P.C.(Alternatives, Possibilities, Choices)}

This strategy helps to generate different aspects that will help a person do things differently. It is an attempt to explore new alternatives, possibilities and choices that can change a situation rather than limiting oneself to obvious or more traditional options.

\subsection{C \& S (Consequences and Sequel)_}

This is a tool for looking ahead to see the short, medium and long term consequences of a potential decision. This tool will help an individual in decision-making as one has to consider all implications on client outcomes.

\section{Aim of the Study}

The aim of this study was to establish: 
i) was there an impact of the critical thinking module on the achievement of the posttest scores between the experiment and the control groups?

ii) how was critical thinking practiced in the delivery of health talks after having attended the critical thinking module?

\section{Research Methodology}

This is a combined quantitative and qualitative study in which a quasi-experiment group design was used to establish the presence of critical thinking of 2 groups of nursing students via a pre-post test (Sowell, 2001; Punch, 2000). To compare the test results between the 2 groups of students, the static-group comparison design was used whereby both groups of student nurses were pretested after which the treatment was instituted to the experiment group.

\subsection{Sampling}

The sample of quantitative approach for this study comprised of 2 groups of students, one being the control group while the other was the experiment group. Each group was made up of 42, year-2 nursing students. These students had already been given the theory of health education and exposed to the clinical experience of carrying out health education in year 2. Their ages ranged between 19 and 26 years. They comprised of $86 \%$ of Malays, $1 \%$ Chinese, $7 \%$ Indians and $6 \%$ other indigenous groups.

While qualitative methods as suggested by Patton (1990) were used to extend a more in-depth understanding of an experience. To achieve that, interviews and health talks was used in this study. Semi-structured questions related to the training module were prepared as a guide to ensure that the interview was more focused. Two sessions of interviews each with semi-structured questions were carried out with 5 students in a conducive environment using a standard interview protocol. The first interview was to gather information and obtain feedback related to the critical thinking module. The second interview was to get their opinions and feedback as to the effectiveness of the module in practice after they had each conducted a health talk to their patients.

\subsection{Instrument}

The research instrument of this study consisted of five vignettes using nursing scenarios related to the main chronic illnesses in Malaysia (Materia Medica Malaysiana, 2005) which were selected for the pre-test and post-test. The selected scenarios for health education management was focused on the areas of heart, pulmonary, endocrine and neoplastic diseases as these conditions are among the eight leading chronic illnesses in Malaysia (Materia Medica Malaysiana, 2005). Each vignette consisted of 9 multiple choice questions formulated in relation to Paul's eight elements of thoughts (1993) for each scenario. Each question was used to assess the underlying thought processes involved since the assessment was not on the answer alone (Nitko, 1996). These vignettes were developed to evaluate the student nurses' abilities to analyze simulated data, identify additional data needed, decide on all possible problems in the scenario, identify nursing interventions and provide a rationale for their responses, hence providing the opportunity to assess the decisions made and the thought processes that were used to arrive at those decisions. Each question consisted of 2 parts. The first part of the question required the student to identify the thought in relation to the given scenario while the second part required the rationale for giving rise to that thought. As this study aimed to establish the presence and the rational of thoughts parallel to Paul's eight elements of thoughts (1993), the student had to select the best answer appropriate to the scenario given.

While for the qualitative part, the semi-structured questions for the interviews were adopted and adapted from the interview format by Teik (2003). Although the questions were arranged and were fixed, the manner and sequencing of asking the questions were rearranged and modified to obtain more information.

\subsection{The Instructional Strategy}

The critical thinking module was delivered in the form of 3 instructional strategies. The first instructional strategy was context-free and was designed to introduce students to critical thinking focusing. The second instructional intervention used a mixed method which taught the students the various thinking tools and at the same time context-free situations or problems forwarded by the student were discussed in relation to the thinking tools learned thus giving feedback to the students to their application. The third strategy engaged the students in a challenging cognitive situation by involving them in applying critical thinking in the teaching and learning process. Reflection was used when students were requested to construct and apply their own critical thinking to the cognitive situations identified.

\section{Findings}

\subsection{Impact of the critical thinking module on the achievement between the experiment group and the control group.}

Table 1 shows the descriptive statistics for the dependent variable (pre-test) by the groups. The score mean of the pre-test of the control group was 62.309 (S.D=7.023), which was almost similar with the score mean of the experiment group, which was 63.357 (S.D=7.560). Before the hypotheses was tested, the difference and standard errors of the post-test scores were calculated between these two groups. The results shows that the mean score of post-test for the 
experimental group is 64.906 ( $S . D=7.140$ ), which is greater than the mean of the control group (Mean $=62.309$; $S . D=8.869$ ). This shows that there is a difference in the score means which is in favour of the experiment group of 2.517. Thus, a t-test was run to determine whether these two groups were statistically significant (see Table.1).

The results from Table 1 shows that the two groups were non statistically significant $(F=0.049 ; p=0.825)$ with $\mathrm{t}$ value -0.257 ( $p=0.789$ ). This result indicated that the post-test score means between the two groups were similar and could be used for further analysis in this research.

In order to examine the impact of the critical thinking between the experiment and control groups on the post-test, the analysis of covariance (ANCOVA) was conducted and the result are shown in table 2. The mean score of the post-test was used as the dependent variable, while the covariance was the pre-test score mean. The results shows that there is a significant difference between the experiment and control groups on the post-test scores $\left(\mathrm{F}_{(1,83)}=10.499, \mathrm{p}<.05\right)$. However, there was no significant interaction effect of treatment and control, on participants' achievement $\left(\mathrm{F}_{(10,83)}=\right.$ $1.418, \mathrm{p}>.05)$.

The results indicate that the treatment gained by the experiment group has an impact on critical thinking achievement significantly compared to the control group. In other words, these results show that there is a significant impact of the critical thinking module on the achievement between the experiment group and the control group (see Table.2). The F tests the effect of group. This test is based on the linearly independent pair-wise comparisons among the estimated marginal means. The results in Table 3 revealed a significant effect of treatments on participants' achievement. The null hypothesis of no significant effects of treatments on participants' achievement was therefore rejected (see Table.3).

\subsection{How is critical thinking practiced in the delivery of health talks after having attended the critical thinking module?}

The students had to prepare the contents and framework of their health talks prior to delivery. As a health talk is an interaction between two or more people, it is often not possible to deliver a health talk according to pre-prepared scripts. Therefore, the students had to be flexible in the manner and sequence of asking questions which may even have had to be simplified in order to obtain, as well as to deliver, important and relevant information. Besides that, questions that were not scheduled for the interview were also included as they were dependent upon responses from the students themselves. Critical thinking, therefore, was carried out by the students when in the process of delivering their health talks to their patients even though they had pre-prepared scripts. This was evidenced through the questions and statements that they made which were guided by the seven thinking tools in the CoRT method.

\subsubsection{Plus, Minus, Interesting (P.M.I)}

The results revealed that the students use PMI to understand patients' knowledge on how they manage their health. It also helps that the respondent showed interest in their history of illness especially their duration of illness as it gave an indication of how their patients managed their disease at home so that remedial advice could be given to them if they were found lacking. An example of the students' concerns is presented below by Student 1 with her patient:

\section{Student: How long did uncle have diabetes?}

Patient: Five six years already but very mild...control only...control...a little control.

Student: I have read uncle's notes just now. Doctor wrote that uncle has taken medicine before?

Patient: Herbal medicine

Student: Like what uncle has told me...you had a small wound before?

Patient: Put lotion and take pills, injection...It was OK

Student: After that uncle did not go and see the doctor?

Patient: Correct...not yet ...felt painful, painful for a few days...admitted direct to hospital...it was a little while only.

Student: This shows that uncle does not understand...your wound and all...because with a wound like this, uncle, we don't put any medicine on it...

Patients with chronic diseases such as diabetes and asthma had yet to come to terms with their disease conditions. The students discovered that only 2 of the 5 patients were still working while the other 3 were unemployed due to their diseases. The students perceived that being unemployed tends to aggravate their patients' feelings of uselessness as expressed by the patient of Student 1 when he said:

"Ar....not working, staying at home only..."

The patients were perceived to be negative and pessimistic towards the course and prognosis of their disease. Discovering that, the students had attempted to motivate them through positive advice and even family duty and obligations as presented below: 
"Uncle is really lucky...to have a wife who is helpful...uncle has to return her kindness, have to look after your wound because if not, the wound becomes like this...difficult for auntie”. (Source: Student 1)

"Have to strengthen your will power...don't eat food that has sugar" (Source: Student 4)

By asking patients to share positive, negative and interesting aspects of their disease management, the students were able to motivate their patients to encourage them to accept their disease in a more positive light. Any new information discovered from the interviews could also be used as a basis for further medical interventions for the patients.

\subsubsection{Consider All Factors (C.A.F)}

Considering all factors involved in a patient's situation and avoiding those that first come to mind is important in order to gather as much information as is possible before a decision is made regarding patient management. Among the factors discussed during their health talks that could bear an influence on the course of their disease included their lifestyles, the impact of social practice such as fasting during the fasting month, food taboos and their gradual sensory loss as presented below:

"Uncle's lifestyle with food is important because it influences uncle's sugar levels in the blood. During the fasting month, uncle does not eat?” (Source: Student 1)

"If we live in the village...there are many things...so easy to get foot injuries...for diabetic patients, auntie, they lost their sense of touch...” (Source: Student 2)

“...our lungs are a little weak...so when we drink...have to be careful a little...when we eat and drink...like don't take so much ice...” (Source: Student 3)

The student nurses had been able to consider the various individual patient factors which exerted a direct influence on their health care management at home.

\subsubsection{Other People's Views (O.P.V)}

It is necessary for the nurse to obtain other people's viewpoints regarding their patients' diseases, especially the significant others in the patients' families as it would indicate their involvement in the patients' care at home as presented by the patient of Student 1:

Student: Who prepares your food at home, uncle?

Patient: The person in my home

Student: She did not cook separately for uncle because she has to sweeten the food?

Patient:She makes the same...she follows my way. If the blood is diluted, she dilutes...did not make separately. That's all.

\section{Student: Family members also drink diluted?}

Patient: If the thing cannot be eaten,(for me ) she will eat it.

Student: Means uncle's wife controls uncle's food

Patient: Yes...she's like that, I admit, not to hide (from you)...she will inform the doctor if she can...

The disruption of household routine can be severe and involves the family doing things for the patient that are observable as well as give rise to negative feelings. The patients' accounts of their families' involvement in their care gave one an idea of their disease management in the home setting. Therefore, if the family perceives the patient's illness in a negative light, the nurses would have to discuss and counsel family members in this respect. This is especially so since the usual health education talks are delivered according to the nurses' perceptions rather than their patients' and their families' needs.

\subsubsection{First Important Priority (F.I.P)}

The nurse has to assess and select priorities when delivering health talks. One cannot assume that all patients have the same needs and therefore have the same priorities. The most important ideas have to be considered first for each individual patient. These may include advising the patient not to self-treat or even emergency measures as presented below:

"Even if it is a small wound, we go and see the doctor. After this, do not put any medicine first. Yes...uncle?" (Source: Student 1)

"OK...sometimes so much work until not eating. Put some sweets ready in your pocket” (Source: Student 2)

“...If feel want to blackout...uncle make some sweet drink...” (Source: Student 4)

Each patient condition requires different interventions and therefore nurse priorities for individual patients should be set according to the individual patient context. 


\subsubsection{Aims, Goals, Objectives (A.G.O)}

Once the priorities have been set for the individual patient, the nurse would have to determine the specific aims, goals and objectives to deliver the necessary interventions to achieve those priorities. Some priorities are in the form of teaching preventive measures and giving information. Some examples are presented as follow;

“....Before uncle sleeps...look all over uncle’s feet...if difficult to look all over...ask your wife or children to look all over, in between the toes...see if there's any injuries...also if you can...wash your feet with warm water (Source: Student 1)

"Sister knows the cause of asthma? Has the doctor ever tell you why asthma?” (Source: Student 3)

"OK...what is your aim for your health? Like your aim...now your asthmatic problem is getting worse right? So how to control control... you take any medicine to control it” (Source: Student 5)

The patients' priorities will determine the specific goals of nursing interventions in meeting the patients' health education needs by providing the relevant information and advice regarding their care at home.

\subsubsection{Alternatives, Possibilities, Choices (A.P.C)}

Knowledge about the consequences of a behaviour is important in making decisions and choices about health behaviour. Different aspects are generated to explore new alternatives, possibilities and choices that could be used to manage a disease. If this aspect is not explored, then health education talks are not complete as these alternatives are already being practiced by most patients in our multi-cultural society even though they may not be officially sanctioned by the doctor. Therefore the patient is open to many options including the option of going for traditional treatment, self-prescribing and self-treating and even the option of not seeking medical help like some of the questions probed by the student nurses below;

"After that uncle did not go and see the doctor?"

(Source: Student 1)

“...did you take traditional medicine? Like medicine from the village?”

(Source: Student 2)

Student 4 also discovered that her patient had a peculiar way of seeking traditional treatment which could be dangerous as his method of treatment was haphazard and gave the patient a false sense of security in not seeking hospital treatment. This is presented below;

Student 4:...Ah...uncle takes any other medicine besides hospital medicine for diabetes?

Patient: Traditional medicine...

Student 4: Traditional medicine?

Patient: Herbs

Student 4: What's that...a drink?

Patient : Drink

Student 4: Medicinal drink...like herbs...

Patient: Yes

Student 4 :How long uncle has been taking it?

Patient : Not often...see if there is any exhibition...

The nurse should not assume that the patients have no other options except to come to hospital for treatment. Since Malaysia is a pluralistic country, it is rich in tradition and social practices. The patients from the various cultural backgrounds often practice a two-prong approach in the management of their disease. Besides following the western type of treatment in hospital, more often than not the patients also fall back on their own traditional health practices for the treatment of their disease. An exploration of this aspect is important to avoid any conflict in the implementation of both approaches to care.

\subsubsection{Consequences and Sequel $(C \& S)$}

The nurse uses this tool to help patients look out for the short, medium and long term consequences of their disease. This would help in prevention efforts as well as to avoid any complications in their disease management at home. Some examples from Student 1 are presented below;

"Like what uncle told me...you have a small wound before?"

"But uncle told me the wound was small...maybe wear shoes?"

“...Uncle put medicine, close up the wound...wear shoes...”

"This shows uncle doesn't understand la...all wounds...because if the wound is like this uncle cannot put in any medicine...” 
"For normal people a small wound can heal but difficult if diabetes... because that place blood supply cannot reach...then later it becomes worst..".

"But because uncle has wound at this foot maybe shows didn't look after feet..."

The other students also warned of the consequences of diabetes if care was not taken:

“...But if a lot of sugar, auntie's sugar level goes up and down also dangerous...If goes down, auntie will feel want to blackout...blackout ...all sweating, sweating...” (Source: Student 2)

"Usually if there's some water in between the toes it will grow microorganisms...that will cause infection" (Source: Student 5)

The outcomes of a disease were discussed during the health talks to make the patients aware of the consequences if care was not taken when managing the disease at home. This would help in reducing the number of complications arising from mismanagement of a disease and indirectly reduce the number of readmissions to hospital. However, on the other hand, even if patients do have an understanding of the disease, it does not gurantee that they will follow proper health behaviours. When delivering health talks, the students had considered and had applied the seven critical thinking skills of the CoRT method where appropriate resulting in the students delivering a more comprehensive health talk tailor-made to the individual patient need.

\section{Discussion}

The results indicated that the critical thinking module, to a certain extent, had a positive impact on the experiment group in helping them to organize their thoughts more comprehensively. This could be due to the fact that those in the experiment group had more exposure and experience in giving health talks to patients as compared to those in the control group although both groups of students had been given the theory on health education while they were in semester 3 (Document 3, Detailed Educational Programme, 2004). Thus, thinking skills need to be nurtured as the skills to think about issues and problems do not suddenly appear in the students (Tama, 1989). Hence, it is assumed across the board that the experiment group of this study was more critical in their thinking capability. Maturity is another factor to be considered as $10 \%$ of the students in the experiment group were older then the control group. Maturity in age constituted a disposition to think critically (Facione \& Facione, 1992) and Alfaro-LeFevre (2004) too agreed that those in the older age group were better thinkers as they had better moral development and more opportunities to practice reasoning in different situations.

Through the health talks that were delivered to their patients, the 5 students had shown that, to a certain extent, they had applied thinking skills from the CoRT method which they had learned when they were given the critical thinking module. By asking their patients on the positive and negative aspects of their disease conditions, the students had an idea of how their patients managed themselves at home. This would help the students determine their patients' priorities as well as their own priorities in helping to fulfill their patient's immediate health needs such as remedial advice which could be given to them if they were found lacking in this aspect. Alder, Porter, Abraham and Teijlingen (2004) had considered this type of situation as a doctor-centred client care as opposed to a patient-centred approach. This is because by asking patients to share positive, negative and interesting aspects of their disease management, the students were able to understand and motivate their patients in order to encourage them to accept their disease in a more positive light. Any new information discovered from the interviews could also be used as a basis for further medical interventions for them.

\section{Implication of the Research}

One of the nurse's functions is to help patients learn and incorporate positive health-related behaviours into everyday life. Helping patients develop new behaviours regarding their health and lifestyle requires considerable expertise from the nurse which is lacking as most nurses have been inadequately prepared resulting in health education activities being carried out in a haphazard manner. Health education is limited to just mere rote-learning in that they repeat to their patients whatever standard health information specific to their disease is required.

The traditional approach to health education emphasizes mainly on physical aspects of ill-health which is reflected in the information base used which represents a highly limited medical perspective. The information is provided in the expectation of an orderly sequence from knowledge through attitudes to "correct" behaviour since human beings are rational beings. However, other social factors such as a person's freedom to choose their health related behaviour are not considered (Downie, Tannahill \& Tannahill, 1998).

Therefore, this could have resulted in health education being perceived by the nurses as an activity separate from routine care which is of little significance. The lack of skills needed for teaching also contributes to haphazard efforts in delivering effective health education. Pre-prepared standardized teaching plans on the various disease conditions which may be outdated, are being delivered to patients on an ad hoc basis prior to discharge from hospital and do not cater to individual patient needs. Nurses do not have to empathize from their patients' perspectives since they have these 
standardized scripts to read from. Once these "health talks" had been delivered, the nurse would consider herself to have carried out her health education nursing function successfully and the various factors such as family involvement, the patients' socio-cultural background and health beliefs, which are crucial in promoting patient compliance, would not be considered or were overlooked. If the nurse does not discuss the treatment regimen in the context of the patient's lifestyle, then according to Patterson, Thorne and Dewis (1998), the teaching is virtually useless.

The nurse teachers should realize and accept the fact that they have among others, the expert power, resource power and position power, to exert influence upon their students who are going to be future trained nurses of the country. Therefore, they play a very important role in the area of teaching health education to student nurses. However, these nurse trainers should be themselves knowledgeable and practioners of critical thinking. Understanding the theory of critical thinking would allow the nurse teacher to have a better perception of an argument as well as provide the nurse teacher with a foundation for explicit guidance and feedback on her students. The better the understanding, the more effectively the teacher will be able to think critically. If carried out often enough, arguments can be handled automatically and pervasively reinforcing his or her beliefs in critical thinking, as the key to mastering the skill of critical thinking is constant deliberate practice.

\section{Conclusion}

The use of critical thinking skills is essential when delivering health education to patients. The thinking skills that general nursing students have are inadequate to deliver effective health education for patients. Findings from this study indicated that though nursing students have their own thinking skills, it is not enough for them to give effective health talks. The critical thinking skills imparted to nursing students through a critical thinking module were practiced and applied when delivering health education. While consensus is agreed by all that there is a need for critical thinking among nursing students. The need for continued efforts and research in this area is evident.

\section{References}

Alder, B., Porter, M., Abraham, C. \& Teijlingen, E. (2004). Psychology and Sociology Applied to Medicine, Edinburgh: Churchill Livingstone.

Alfaro-LeFevre, R. (2003). Critical Thinking in Nursing: A Practical Approach, 3rd Edition, Philadelphia: Saunders.

Alfaro-LeFevre, R. (2004). Critical Thinking and Clinical Judgement: A Practical Approach, 3rd edition, St. Louis: Saunders.

Bandura, A. (1989). "Social Cognitive Theory", In R. Vasta ( Ed.). Annals of Child Development. Vol. 6. Six theories of child development (pp1-60) Greenwich, CT: JAI Press. [Online] Available: http://java.cs.vt.edu/public/classes/comminites/uploads (6 April 2008).

Chua, S. L. (2004). Nursing and Midwifery Board Malaysia. [Online]. Available: http://medicalprac.moh.gov.my (23 May 2007).

De-Bono, E. (1999). Six Thinking Hats, Suffolk: First Back Bay.

Document 3, Detailed Educational Programme, (2004). 4th edition, Kolej Perubatan DiRaja, Perak.

Downie, R. S., Tannahill, C., \& Tannahill, A. (1998). Health Promotion: Models and Values, 2nd edition, Oxford: Oxford University Press.

de-Bono's CoRT Programme. (1972). [Online] [Accessed on]. Available: http://www.edwdebono.com/cort/debonocort.htm (20 January 2005).

Facione, N. \& Facione, P. (1996). Externalizing The Critical Thinking In Knowledge Development and Clinical Judgement, Nursing Outlook, 44(3), 129-136.

Jackson, M. (2004). "Defining the Concept of Critical Thinking”. In Smeltzer, S.C., Bare, B.G., Hinkle, J.L. \& Cheever, K.H.(2008). Brunner \& Suddarth's Textbook of Medical-Surgical Nursing, 11th edition, Philadelphia: J.B. Lippincott Company, 178-185.

Kozier, B., Erb, G., Berman, A. \& Snyder, S. (2004). Fundamentals of Nursing: Concepts, Process and Practice, 7th edition, New Jersey: Pearson Education Inc.

Ministry of Health Malaysia. (1996). Document 3: Health Curriculum, Kuala Lumpur.

LeMone, P. \& Burke, K. (2008). Medical Surgical Nursing: Critical Thinking in Client Care, 4th edition, New Jersey: Pearson Prentice-Hall.

Leong, S. L. (2005). A Survey on Home Based Nursing, Journal of Health Education Royal College of Medicine, 2(1), 34-37.

Martin, C. (2002). The theory of critical thinking of nursing, Nursing Education Perspectives, 23(5), 243-247. 
Materia Medica Malaysiana, Malaysians Exposed to Burden of Eight Main Illness Last Year. [Online] Available: http://malaysianmedicine.blogspot.com/2005 $0301 \mathrm{htm}$ (16 March 2005).

$\mathrm{Ng}$-Eng, T. (2003). An Investigation into the Effectiveness of a Merged Mode of Instruction for the Teaching of English Language Existing in a Malaysian Secondary School. Unpublished Master's thesis, Universiti Sains Malaysia.

Nitko, A. J. (1996). Educational Assessment of Students, Englewood Cliffs, NJ: Prentice Hall.

Norwahida, R., Hairne, Y., Abdul, H. \& Nurulaidah, A. (2006). Level of Concern in Taking Medicine among Diabetes Patient, Journal of Health Education Royal College of Medicine, 5(3), 24-33.

Patterson, B., Thorne, S. \& Dewis, M. (1998). “Adapting to and managing diabetes, image”. In Rankin, S. H. \& Stallings, K.D.(2001). Patient Education: Issues, Principles, Practices, 4th edition, Philadelphia: J.B. Lippincott Company, 30-41.

Paul, R. W. (1993). Critical Thinking: "How to prepare students for a rapidly changing world, Santa Rosa, CA: Foundation for Critical Thinking”. In Taylor,C., Lillis, C. \& LeMone, P. (2000). Fundamentals of Nursing: The Art and Science of Nursing Care, 5th edition, St Louis: Lippincott Williams \& Wilkins. 17-25.

Patton, M. Q. (1990). Qualitative Evaluation and Research Methods, London: Sage.

Punch, M. Q. (2000). Developing Effective Research Proposals, London: Sage.

Rankins, S. H., Stallings, K. D. \& London, F. (2006). Patient Education in Health and Illness, 5th edition, Philadelphia: Lippincott, Williams \& Wilkins.

Sowell, E. J. (2001). Educational Research: An Integrative Introduction, New York: McGraw-Hill, Inc.

Tama, M. C. (1989). Critical Thinking has a Pace in Every Classroom, Journal of Reading, 33 (1).

Wilkinson, J. M. (2001). Nursing Process and Critical Thinking, New Jersey: Prentice-Hall.

Table 1.T-test Results of Two Groups’ Pretest Score Means

\begin{tabular}{|c|c|c|c|c|c|}
\hline & \multicolumn{2}{|c|}{ Levene's Test for Equality of Variances } & \multirow[t]{2}{*}{ t value } & \multirow[t]{2}{*}{ df } & \multirow[t]{2}{*}{ sig. } \\
\hline & F value & Sig & & & \\
\hline $\begin{array}{c}\text { Equal Variances } \\
\text { Assumed }\end{array}$ & .049 & .825 & -.257 & 82 & .798 \\
\hline $\begin{array}{c}\text { Equal Variances Not } \\
\text { Assumed }\end{array}$ & & & -.257 & 82.000 & .798 \\
\hline
\end{tabular}

Table 2. Summary of Analysis of Covariance Results

\begin{tabular}{|c|c|c|c|c|c|}
\hline Source & Type III Sum of Squares & $\mathrm{df}$ & Mean Square & $\mathrm{F}$ value & $\mathrm{p}$ value \\
\hline Corrected Model & $346.260(a)$ & 23 & 15.055 & 2.758 & .001 \\
\hline Intercept & 195534.942 & 1 & 195534.942 & 35823.601 & .000 \\
\hline Pretest & 114.728 & 12 & 9.561 & 1.752 & .078 \\
\hline Group & 57.304 & 1 & 57.304 & 10.499 & $.002 *$ \\
\hline Group x pretest & 77.420 & 10 & 7.742 & 1.418 & .194 \\
\hline Error & 327.496 & 60 & 5.458 & & \\
\hline Total & 339806.222 & 84 & & & \\
\hline Corrected Total & 673.757 & 83 & & & \\
\hline
\end{tabular}

Table 3. Univariate Analysis of covariance of the effects of experiment and control on participants' achievement

\begin{tabular}{cccccc}
\hline & Sum of Squares & Df & Mean Square & F & Sig. \\
\hline Contrast & 57.304 & 1 & 57.304 & 10.499 & .002 \\
Error & 327.496 & 60 & 5.458 & & \\
\hline
\end{tabular}

